\title{
MODELS OF THE EARTH'S MAGNETIC FIELD
}

\author{
BY \\ JOSEPH C. CAIN
}

\section{GPO PRICE \\ s \\ CFSTI PRICE(S) S}
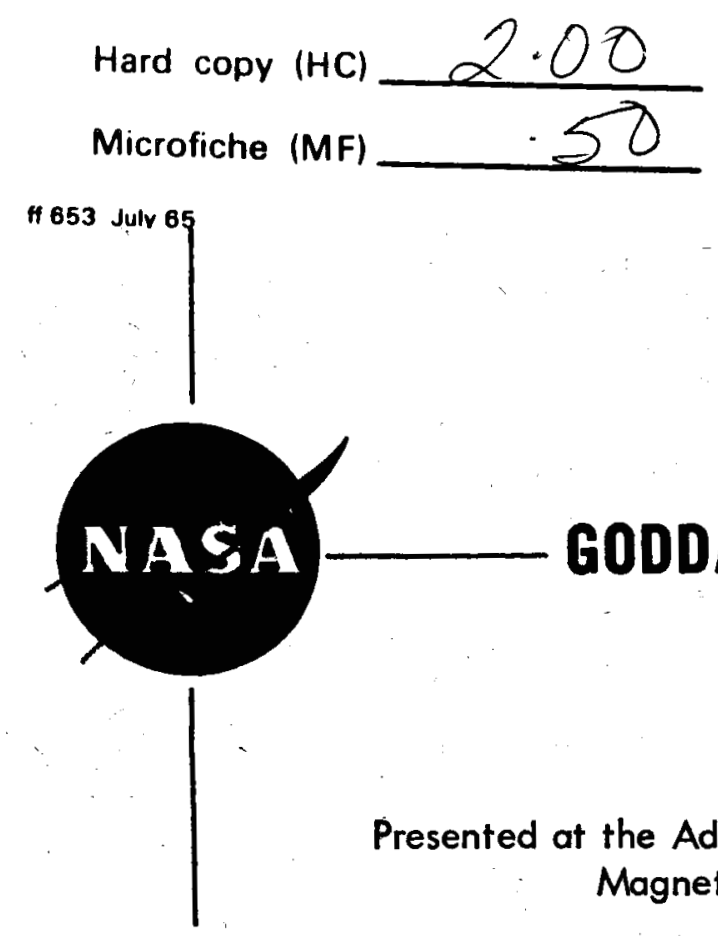

GODDARD SPACE FLIGHT CENTER GREENBELT, MARYLAND

Presented at the Advanced Study Institute (Radiation Trapped in the Earth's Magnetic Field) August 16, 1965 - Bergen, Norway 
Models of the Earth's Magnetic Field

Joseph C. Cain

NASA-Goddard Space Flight Center

Greenbelt, Maryland

October 1, 1965

*Presented at the Advanced Study Institute, "Radiation Trapped

in the Earth's Magnetic Field," August 16, 1965, Bergen, Norway. 


\section{Captions}

Figure 1. Hourly values of the horizontal component of the geomagnetic field plotted verses U.T. for five days in January, 1964. The data were taken from averages of 2.5 minute digitized values for those stations where an * appears. Data for the other observatories were taken from their tables of hourly values.

Figure 2. Positions of magnetic survey observations available for the interval 1945-1964 with some thinning as described in text.

Figure 3. Total field $F$ and plot of external field intercepts using GSFC (7/65) coefficients at earth's surface for 1960.0. External field vector enters at + and exists at 0 with ellipses indicating standard errors of position.

Eigure 4. Standard error of $\operatorname{GSFC}(7 / 65)$ fit computed for total field F (gamma) at earth's surface for epoch 1960.0 .

Eigure 5. Standard error of GSFC(7/65) fit computed for inclination I (degrees) at earth's surface for epoch 1960.0.

Figure 6. RMS deviations between survey data by year and computed fields listed in Table 2. Numbers at end of each curve give RMS over the whole interval 1945-1964. Histogram on lower diagram gives distribution of data per year. 


\section{Introduction}

In order to discuss models of the earth's magnetic field it is necessary to understand the use to which they will be put and the expected range of their validity. The evaluation of experimental data both of the field itself and of charged particles trapped in that field require a detailed and accurate reference field structure throughout the magnetosphere. Theoretical studies are generally not as particular and have frequently subsisted on even a dipole approximation to the main field. However, as our knowledge of the field and particle fluctuations extends outward from the earth to the magnetospheric boundary and into the antisolar tail regions, even the theoretical workers will require a better model than that of the extrapolated dipole.

For most experimental work the dipole approximation is inadequate at any altitude since the main field is very irregular near the earth, and beyond a few earth radii where it becomes more dipolar, the distortions due to other effects become significant. Also, as experimental work on the field itself near the earth becomes more precise, even the relatively small time variations in these regions will need to be considered.

In order to better define the scope of magnetic field models that are in the process of being developed we define here four separate field sources as follows:

1. CORE FIELD - This is the main field from the molten core of the earth as it has historically been plotted on surface magnetic charts of the world and has been analyzed into a potential function using series of spherical harmonic coefficients. Its secular variation 
is characteristically a fraction of a percent per year in intensity and presumably arises from mass and wave motions in the molten core. 2. CRUSTAL FIELD - This additional source of the magnetic field is thought to arise from the magnetized crustal material from the earth's surface to depths of the order to $20 \mathrm{~km}$ where the temperatures rise above the curie point. Its contribution to the ambient field near the earth's surface is frequently large in producing irregularities of a few kilometers in extent and amplitudes of a few thousands of gamma $\left[1 \gamma(\right.$ gamma $)=10^{-5} \Gamma$ (gauss $\left.)\right]$. Measurements of the total scalar field $\overrightarrow{\mathrm{F}}$ by sounding rockets (Davis, et al, 1965) indicate that there are measurable contributions of the crustal field at least to $50 \mathrm{~km}$ altitude. It is hoped that for most geomagnetic work this source may be smoothed over since its inclusion would require an extensive set of parameters.

3. IONOSPHERIC CURRENTS

(a) Quiet daily variation ( $\mathrm{Sq}$ ) including equatorial electrojet

(b) Disturbance variations (DS) including auroral electrojets

\section{PLASMA PRESSURES}

(a) Internal - Trapped particle and tail (?)

(b) External - Boundary

The last two sources, 3 and 4, cannot be discussed without making a distinction between a "forecast" and a "definitive" model of the field. In the past, the world magnetic charts for a given epoch (e.g. 1965) were based on survey data for prior years extrapolated to that epoch. This 
extrapolation of the secular change in the core field was used over a future period of 5 or 10 years and thus is entirely a "forecast" field model. A "definitive" map of the core field, not generally drawn, would instead be based on an interpolation of data taken over roughly equal intervals about some mean epoch.

To extend these concepts to the sources of field distortions external to the earth, we must understand the features of those distortions that are statistically predictable and might be included in a "forecast" model and those that are patently irregular and, except for probability estimates of occurrence, should better be left to "definitive" models.

An example of geomagnetic field variations as measured at the earth's surface is given in Figure 1. These curves are hourly mean values of the horizontal component measured at a number of magnetic observatories and plotted to the same scale but arbitrary baselines. The vertical lines denote $0 h_{\text {UT }}$ between the days indicated, and the position of the dots gives the times of local noon at each station. The interval chosen is moderately quiet and is thus free from large magnetic disturbance. At most of the low latitude stations one can see the regular peak of the Sq variation near local noon. However, contrary to the impression that might be conveyed by statistical treatments of the subject, one can note many day-to-day changes in the data from the lowest latitude stations and increasing irregularities with increasing latitude. For example, the curve for Huancayo shows a noon minimum instead of the usual maximum on January 12. The slow increase of the level of the curves is typical of that following a magnetic disturbance. The average of these 

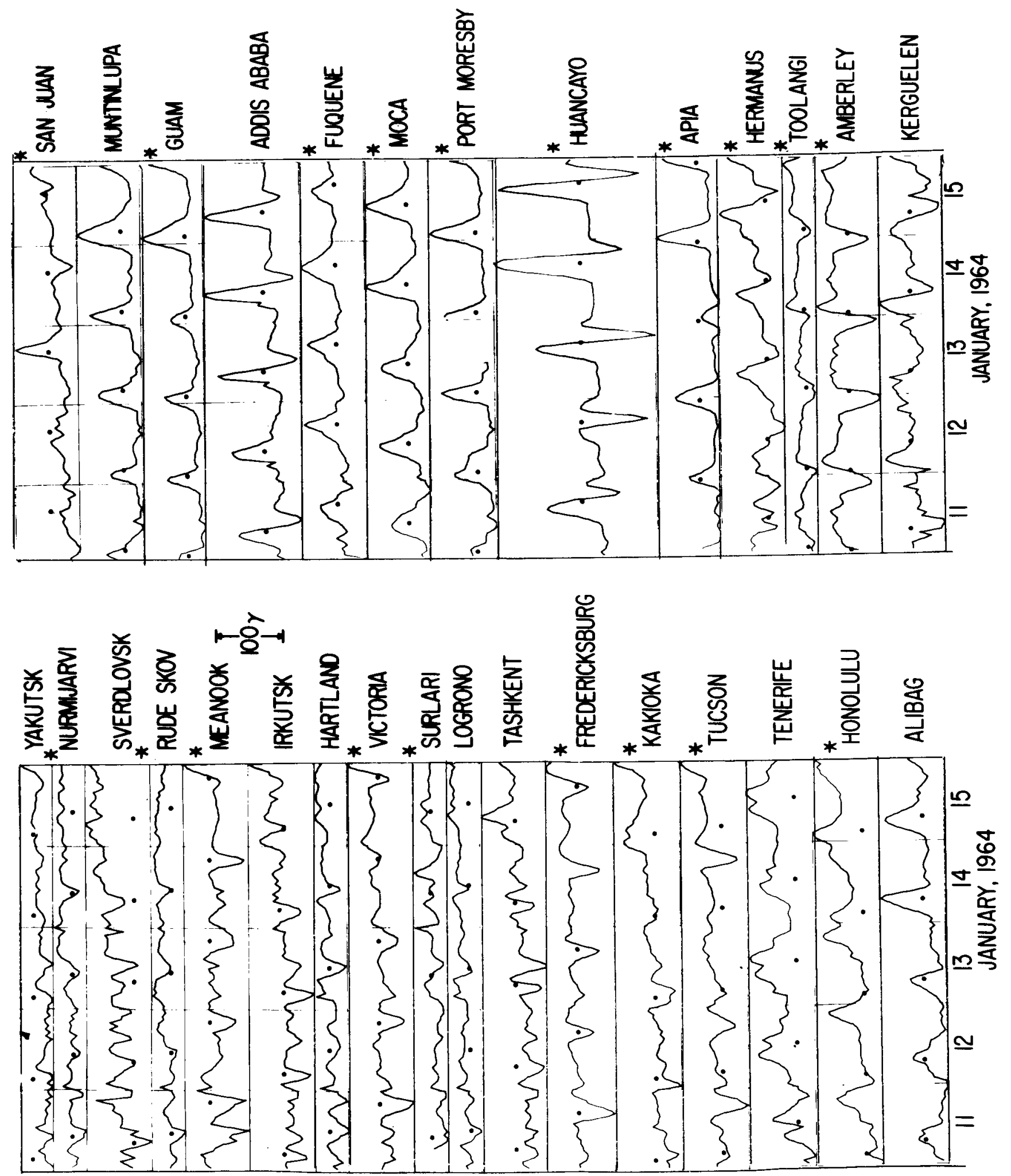
disturbances in the horizontal field over all longitudes (Dst) has been plotted many times in the past (e.g. Sugiura, 1964) and is indicative of the irregular changes in the field with the onset of magnetic disturbance. These disturbances occur several times a year and cause a general lowering of Dst by a few hundred gammas. While the major source of the quiet daily variations Sq are thought to lie in the conducting layer of the ionosphere near $100 \mathrm{~km}$, the Dst variations are speculated as being caused by the expansion of the field by trapped particles. At the present time the distribution of the geoeffective particles is not known nor is it clear that there is a significant steady component ( $\mathrm{cf}$. Hoffman and Bracken, 1965). There is however evidence (Ness, 1965) that some of the trapped plasma is sufficient to completely distend the earth's field in the antisolar direction.

The external pressures from the solar plasma have now at least been grossly defined and we should see later in the symposium evidence supporting the numerical evaluations by Mead (1964) of its effect.

Thus in order to effectively model the field of the earth within the magnetosphere even a "forecast" field will need to include these external sources to some extent. For a field reference for some types of experimental data it may eventually be necessary to use a "definitive" model of the field which is based on the interpolated best estimate of the actual field distortions at a given time using whatever surface and satellite measurements of the field that can be obtained. This type of dynamic field modelling may be somewhat unnecessary for present particle work. It would of course enormously complicate the computation of the $L$ parameter used for organizing the data. 
In order to avoid this complication most of the remainder of this discussion will be confined to the core field and its secular change and thus only cover a fraction of the scope of the subject.

\section{Survey Data}

Before discussing the evaluations of the field it is very useful to note the character of the magnetic survey data currently available. These data are characterized more by their heterogeneity than any uniformity. The sources are many and include data from surveys by 1 and and from magnetometers both on board and towed behind ships and aircraft. The quality of the data varies from some where even the component being measured is in doubt, to the high quality observations performed by some survey groups and generally by the magnetic observatories. The data may be comprised of instantaneous measurements or time averages over periods up to a year. They may be presented either with no modifications, modified to attempt to eliminate diurnal variations, or corrected to a given epoch by spatially interpolating the secular change from magnetic observatories. The observatories, though currently numbering some 300, are almost all confined to the land masses of the northern hemisphere and thus not give a reliable measure of the secular change in most southern areas.

For the whole interval 1929 to 1950 , there were almost no survey observations of the ocean areas. Since that time the U: S. Navy project MAGNET has been the main contributor of ocean magnetic data. However, even with the present increased efforts being made during the World Magnetic Survey, 


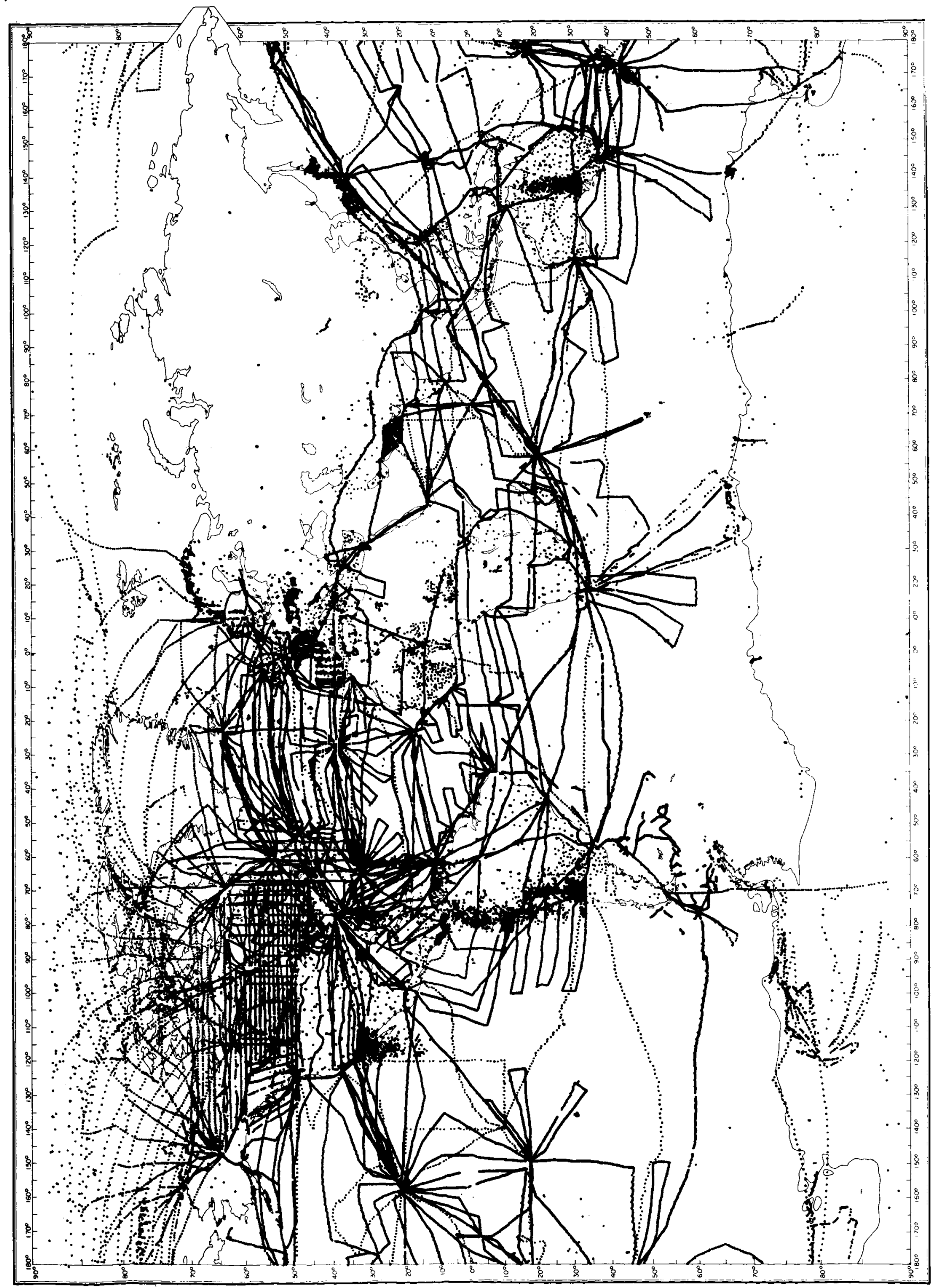


there are yet significantly large areas of the earth covered only by a single survey track or not at all. The ocean gaps include the South Pacific centering near the meridian $120^{\circ} \mathrm{W}$, and the Atlantic and Indian Oceans south of $-40^{\circ}$ latitude. There are also large 1 and areas only sparsely covered such as Asia with only Russian observatory annual mean data since 1940 and the Antarctic with only scattered observations. The altitude distribution of the data is equally heterogeneous with the only presently available satellite data being those from Vanguard 3 and some less accurate $(\sim 100 y)$ data from the Alouette satellite. The Vanguard 3 measurements cover only small volumes in areas near the NASA tracking stations and Alouette covers Canada which is already densely covered by aircraft observations.

Clearly, any method of data analysis may produce results that could be greatly affected by this way very heterogeneous data distribution.

\section{Technique of Analysis of the Core Field}

We have recently completed an analysis of the data available over the period 1940-1962 and reported the results in comparison with most previously available work (Cain et al, 1965). Our approach to the problem differs from that of most past workers in that we have attempted to fit the secular change of the field simultaneously with the spatial structure. The principal used is to minimize the mean square difference between each observed component $(H, D, I, Z, F)$ and a computed component. The field expansion for this work was an internal potential function of the classic form

$$
V=a \sum_{n=1}^{\infty}\left(\frac{a}{r}\right)^{n+1} \sum_{n=0}^{n}\left(g_{n}^{m} \cos m \varphi+h_{n}^{m} \sin m \varphi\right) P_{n}^{m}(\theta)
$$


where $r, \theta, \varphi=$ spherical coordinates corresponding to geocentric radius, geocentric colatitude, and longitude. The coefficients $g_{n}^{m}, h_{n}^{m}$ are gauss coefficients $\left(g_{\mathrm{I}}^{\mathrm{O}} \approx-\cdot 3\right)$ and $\mathrm{P}_{\mathrm{n}}^{\mathrm{m}}(\theta)$ are Schmidt's quasi-normalized polynomials. The various field components are evaluated using $\overline{\mathrm{F}}=-\nabla \mathrm{V}$ on the assumption that $\nabla \times \bar{F}=0$ (no current density inside the region of interpolation)

The minimization procedure deviates from a straightforward leastsquares only in that each component is expanded into a Taylor's series in the coefficients and, starting with a first approximation to the field, the solution is found for the first order corrections to the coefficients. This procedure is necessary since the fit is made to the components $D, I, H$ and $F$ which vary non-linearly with the coefficients.

In order to include the secular change, the coefficients themselves are expanded in a power series about the mean epoch of the data and the first time derivatives determined along with the coefficients themselves.

In performing the fits it has been found that more consistent results can be obtained by taking into account the earth's oblateness. Thus, whereas the value a in the expression for $V$ is arbitrarily taken to be the mean radius of the earth $6371.2 \mathrm{~km}$, the value of $\underline{\mathrm{r}}$ used for each data point assumes the surface data on the Kaula ellipsoid (equatorial radius of $6378.165 \mathrm{~km}$ and a flattening factor of 1/298.3). In addition, the slight $\left(\sim 0.2^{\circ}\right)$ rotation of the geocentric verses geodetic direction is taken into account. In computing the field from the derived coefficients a precise evaluation needs to allow for both of these factors. Assuming that the geodetic latitude is $\lambda$ so that the rotation angle is $\delta=\lambda+\theta-90^{\circ}$, the measured magnetic components are: 


$$
\begin{aligned}
& X=-F_{O} \cos \delta-F_{r} \sin \delta \\
& Y=F_{\varphi} \\
& Z=F_{O} \sin \delta-F_{r} \cos \delta
\end{aligned}
$$

where $F_{O}, F_{\varphi}, F$ are the three geocentric components of $\bar{F}$. One should also note that although the coefficients are derived using Schmidt normalized polynomials $\mathrm{P}_{\mathrm{n}}^{\mathrm{m}}(\theta)$, an internal conversion is normally made in the FORTRAN computer codes (Cain et al, 1964) so that the coefficients actually used correspond to the Gauss-Laplace functions $P^{n, m}(\theta)$. This conversion is useful in that the number of computer operations necessary for the evaluation of the field components is reduced by about $10 \%$. A New Test Model of the Core Field

Before evaluating the relative merits of existing models of the core field we would like to report on an additional model that was recently derived using improved techniques and some added data. This model, which we will here label GSFC(7/65), according to the organization and date of derivation, was intended as a test to see whether higher order spatial coefficients and a simple external field are statistically significant if almost all of the available data are used. Since we have recently published an evaluation of the field (Cain, et al, 1965) we will discuss here only those details which differ from the prior work.

One change in the techniques of computation that allows a significant expansion of the number of coefficients over the previous analysis is the use of single precision ( $\sim 8$ digit) arithmetic in the formation and solution 
of the matrix of values entering the equations for the normal equations. The previous use of double precision arithmetic $(\sim 17$ significant figures) was learned to be helpful in some very special circumstances, but was found not necessary for general use (Leaton, 1964). The error in the final parameters resulting from single precision arithmetic is estimated at less than $10^{-5}$ of their value.

In order to test the use of higher order coefficients, the number of spatial parameters was expanded to 99 (maximum $n=m=9$ ) and first time derivatives to $48\left(\dot{n}_{m}=6\right)$, although in the actual analysis to be described here an $\dot{n}_{m}$ of only 5 was used. In addition to these internal sources, a simple external field was added. In keeping with most theoretical studies that predict that any external source of field would result in a parallel flux across the earth, the form of the external field was taken to be three orthogonal components $E_{1}, E_{2}, E_{3}$ where $E_{1}$ is northward along the geographic axis and $E_{2}$ and $E_{3}$ are in the equatorial plane directed respectively at geographic longitudes 0 and $+90^{\circ}$.

Following the suggestion of Kahle, Kern and Vestine (1964) for a checking term, we initially also included the term $\dot{\mathrm{g}}_{0}^{\circ}$. (The formulation thus as sumes $\nabla \times \bar{F}=0$, but $\nabla \cdot \overline{\mathrm{F}} \neq 0$ !)

Since we also wished to test the differences to be obtained by the use of most of the available data, only a small amount of data deletion was done. The data for the period 1945-1964 were grouped into categories of surface, aircraft, observatory, and satellite. The data set used comprised all observatory and satellite (Vanguard 3 and Alouette) data in this interval. 
The surface and aircraft data were then each "clipped" only in areas of $2^{\circ}$ latitude $\times 2^{\circ}$ longitude where the density exceeded about 100 observations per $10^{5} \mathrm{~km}^{2}$. That is, one observation per $2^{\circ} \times 2^{\circ}$ block at the poles was allowed and used as a maximum density for the lower latitude blocks. The resulting data distribution of some 197,000 component and total field observations is given in Figure 2. The previously discussed "sparse areas" become very evident.

One other difference in this analysis concerns the weighting of the data as entered into the normal equations. Previously, the observatory annual means were weighted by a factor of the order of 6 to 10 over other survey data according to their high estimated accuracy. In this analysis the weights were arbitrarily reduced so that they were only of the order of 2-4 times that of the other survey data since it was felt that the previous weights were too high. It is yet unclear as to the improvements in the derived fields when the data are weighted inversely according to their estimated errors.

Test runs were first made on random samples of the data, skipping to each nth observation, to test the significance levels and ascertain whether there were any spurious correlations between the coefficients. This was the first time that the correlation coefficients have been derived between the various parameters. It was gratifying to find correlation coefficients between all of the parameters of only a few tenths and hence practically insignificant. This result was especially reassuring since it is clear 
that neither the external terms nor the first time derivatives of the spatial terms have any theoretical reasons for being orthogonal over the earth's surface. As the tests were made first starting with every 200 th observation and their increasing the number of observations to only skip to every 100 th, and then to every 50 th, it was noted that the absolute values of both the $g_{0}^{0}$ and the E terms continuously decreased and were always comparable with their estimated standard error. However, when enough data were added to bring the standard error of the external field E down to about 30 y the components began to stabilize and assume fixed values. However, the $g_{0}^{\circ}$ term continuously decreased and was deleted when both its absolute value and standard error were of the order of $8 \gamma$. It is thus established that if there was any monopole component to the earth's field (indeed, if monopoles existed!) that its level would need to be less than $8 y$. This behavior in relation to the estimated standard errors does give some indication that whatever the cause, the external component of the earth's field is a statistically significant aspect of the data set. The resulting set of harmonic coefficients is given in Table 1 to $n=m=9$ in the spatial terms, $n=m=5$ in the first time derivatives, and the three external terms. The estimated standard errors for the external terms and the low order spatial terms range from 4 to $5 y$ reducing to a $2-3 y$ level above $\tilde{n}=6$. The standard errors of the time derivatives are of order ly/ year.

A comparison of the present coefficients with our most recently published set ( $\underline{\text { Cain }}$ et $\underline{\text { al }}, 1965$ ) shows good agreement, though for the 
TABLE 1

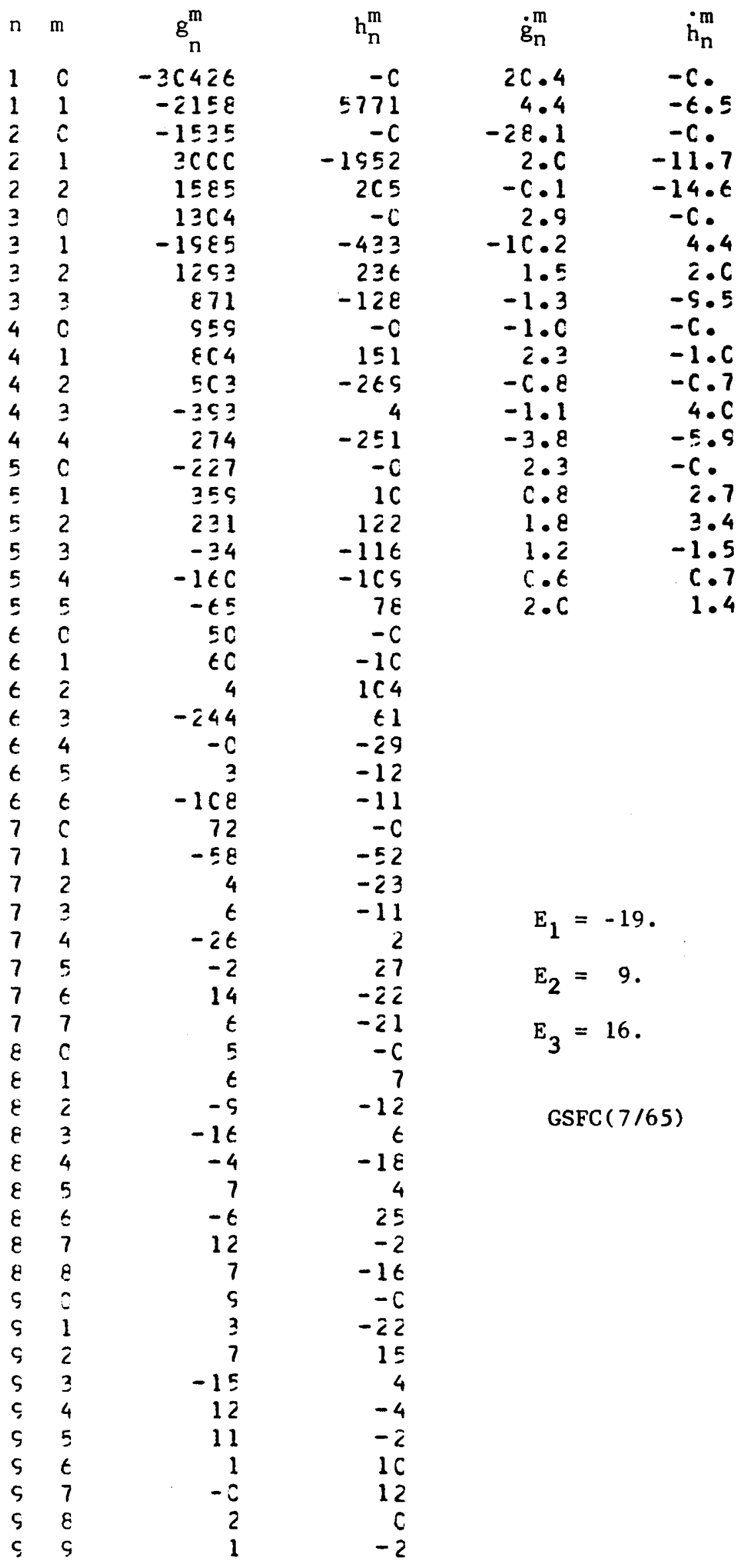


Table 2

Characteristics of Four Models of the Core Field

Field

$\mathrm{J}+\mathrm{C}$

GSFC (64)

LME

$\operatorname{GSFC}(7 / 65)$
Number of Coefficients spatial

48

$0 *$

1960

35

63

80

48

35

99 internal

+3 external
Epoch

Derived from
1965

$7400 \mathrm{H}$ and $\mathrm{F}$ Observations 1940-1960. *First derivatives were actually derived but not published. (Jensen and Cain, 1962)

196021695 selected observations 1940-1963 (all components) (Table 4 from the paper by Cain et al, 1965)

aircraft and surface observations converted to $X$ and $Y$ components and corrected to epoch 1965 using separate analysis of observatory secular change (Leaton, Malin and Evans, 1964)

197,000 selected observations 1945-1964. 
spatial terms too frequently outside their estimated standard errors. This discrepancy can likely be explained by the fact that although the functions are orthogonal to one another if averaged over the whole sphere, they are not orthogonal over the available data set. As more terms were added for the present analysis it might be predicted that there would be some adjustment of the lower order coefficients as the still neglected higher order harmonics distribute their aliases differently. Some differences are also likely since the data set extends over the period 1945-1964 including some newer data whereas the last fit took an average over the period 1940-1962. As shown in the previous paper the secular changes in the field are not completely linear even over a 20 year interval.

One of the new results of this analysis is the presence of a statistically significant external field. However, even with the estimates of its standard error it is still not conclusive that it is factual due to the poor distribution of data. A plot of its orientation relative to the surface field contours of $\vec{F}$ is shown in Fig. 3. Here the + denotes the field entering in the northern hemisphere. If we were to speculate on the possible origin of a constant external field we should presently need to turn to one of two effects, both of which would result in almost uniform fields parallel to the earth's axis. The effect of the compression of the earth's field by the solar wind should give a northward flux, whereas the effect of trapped plasma should expand the field to give a net decrease as presumably is enhanced during the main phase of a magnetic storm. The fact that the 


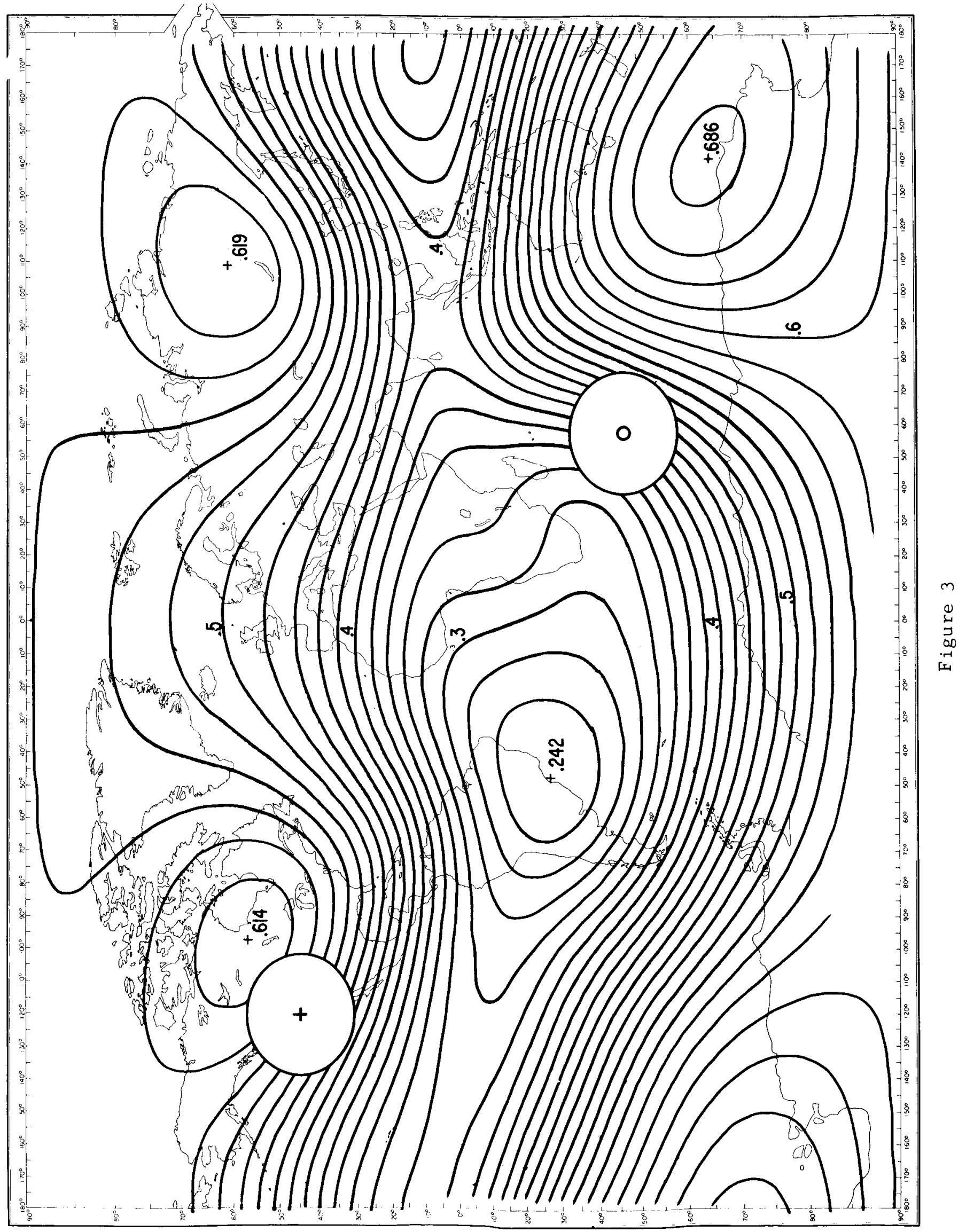


observed constant external field has such a large equatorial component is puzzling and leads one to believe that perhaps it is a spurious result of the irregular data distribution or some other unknown factor. However, since it is at least in the correct quadrant of the earth to be associated with the eccentric dipole, the result would imply that the net internal plasma pressures more than balance the external to produce a net uniform flux which would weaken the field near the equator.

\section{Spatial Errors of Derived Field}

Although it is not possible to know with certainty the absolute errors of the fits, it is possible to obtain some statistical measure of how much the potential function might be varied by moving all of the data through one standard error. Such a spatial function can be derived on the assumptions that the standard errors of all of the data are equal to that of the fit and that all are independent. A resulting spatial map of the standard error in total field F (in gammas) is given in Figure 4. The highs and lows in the field are marked by $H$ and $L$ respectively along with their values in gamma. A comparison between this figure and Figure 2, the data distribution, shows as might be expected, that the fit could be more easily altered in the regions where the data are sparse. However, although this map gives the possible relative errors in the fitted field, it is likely that the actual errors of the field are several times this. This assertion is based on sample comparisons between measured and computed field values which indicate that there are frequent systematic variations of the order of a few hundred gammas which are not due merely to the crustal anomaly "noise" (cf. Figure 6 in our 


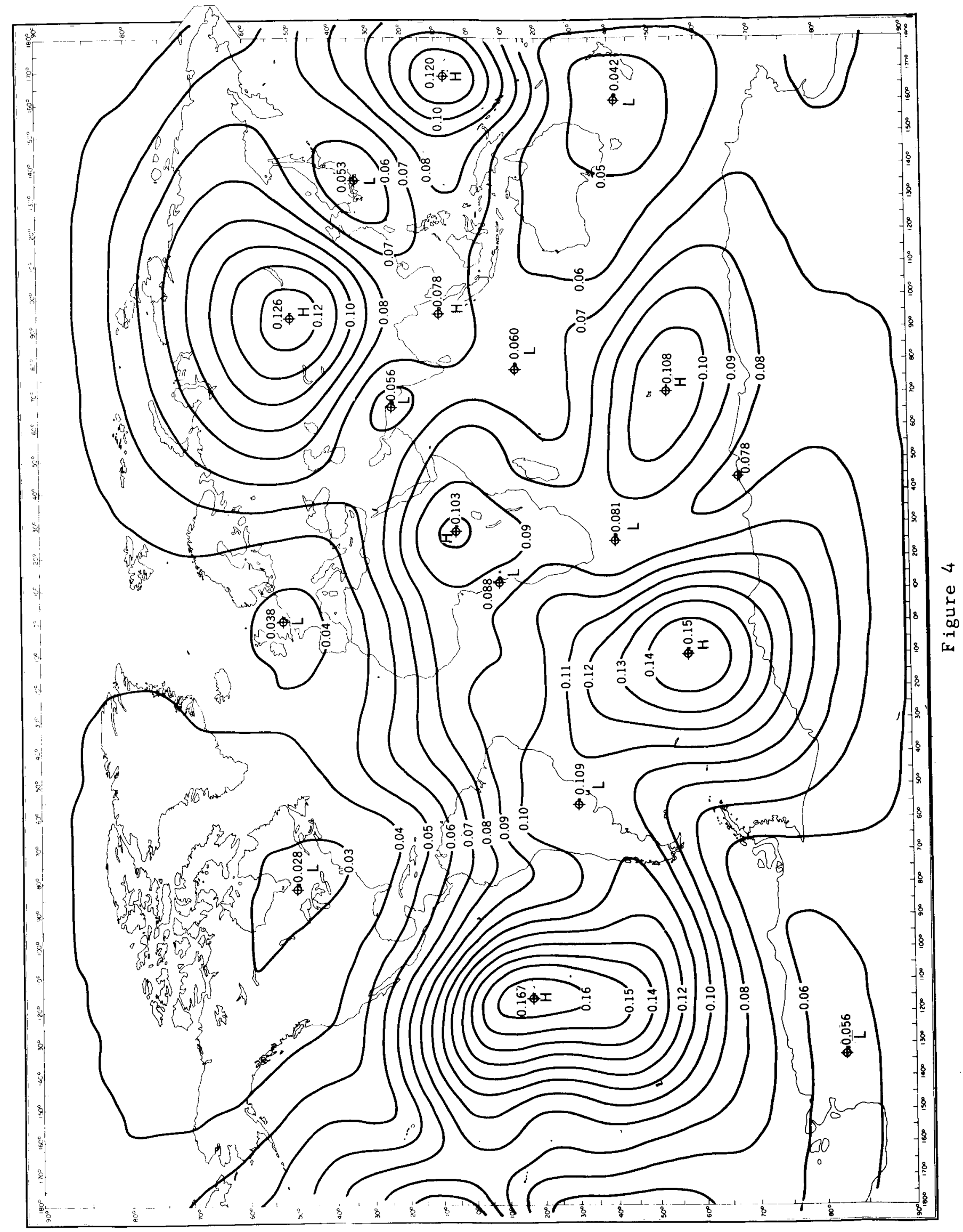




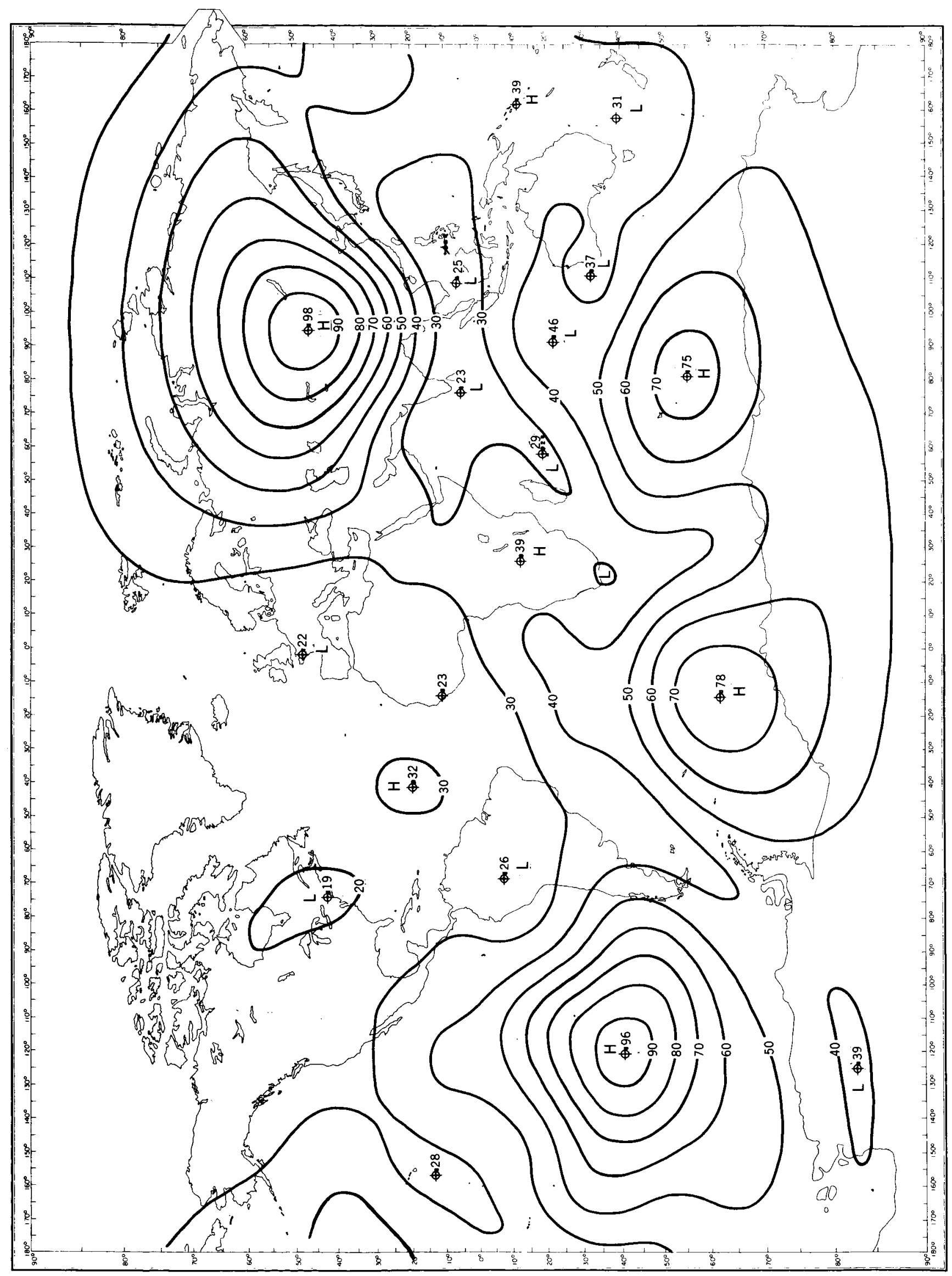

$\underset{0}{0}$ 
previous paper [Cain et al, 1965]). The corresponding standard error map of inclination is given in Figure 5 and shows a range of a factor of four or five between the flexibility of the fit in the sparse data areas to that in the better surveyed regions.

It is useful also to investigate the standard error estimates in regions outside the data volume both with increasing altitude and at years other than those near the mean epoch. Sample calculations of the errors with altitude show that although the $\Delta \mathrm{F}$ starts at differing values at the surface it tends to decrease to a constant level of the order of 15-20y by $2000 \mathrm{~km}$ altitude and thereafter to remain constant at about 10-15ץ. When the errors are computed for epoch 1965 instead of 1960 they increase by about 30-50\%. By 1970 the estimated errors are approximately double those computed for 1960. It is thus likely that any definitive field will always have significantly smaller errors than any forecast field where the latest data lag by about two years. If future (satellite) data are processed quickly to reduce this time lag, the errors in a forecast field should be correspondingly smaller. It has been learned by use of fewer coefficients in the fitting process that whereas a smaller coefficient set gives a larger overall standard error in regions of high data density, the error in regions of low density are almost the same as those shown in Figures 4 and 5 . Of course if the number of coefficients was increased still more so as to include those with little statistical validity it is likely that the possible variation of the fit in the areas completely void of data could be increased significantly. 
The conclusion of such studies on the present data set is that whereas the number of coefficients is indeed larger than most derived in the past, this number seem necessary to fit the data and that no significant harm has yet been done in causing large errors in the areas of low data density. If anything, it will be enlightening to expand the coefficient set further to see whether we have reached the optimum number of coefficients necessary to describe the present data. It is likely that there are even shorter wavelengths in the available data. However, before making such a coefficient expansion we intend to investigate independently the spatial spectrum to see whether a better overall result might be better achieved by first filtering the data to eliminate any effect from the neglected harmonics. It is also worthwhile to postpone such evaluations to see whether the POGO satellite will provide sufficient data to relieve some of the problems with the data density.

\section{Comparison of Field Models}

The previous paper made comparisons of numerous available core field models with the available survey data. The RMS deviations of all the component observations per year were listed combining such quantities as $(\Delta F)^{2},(\Delta H)^{2},(F \Delta I)^{2},(H \Delta D)^{2}$ and $(\Delta Z)^{2}$. We will extend this comparison here and focus on the three best available fields as well as the older $\mathrm{J}+\mathrm{C}$ model (Jensen and $\underline{\mathrm{Cain}}, 1962$ ) now in general use for the determination of the $\mathrm{L}$ parameter for organizing trapped particle data. A comparison of these four fields (see Table 2) is given in Figure 6. Plotted at the top are the RMS deviations from the data for each year and given at the end 

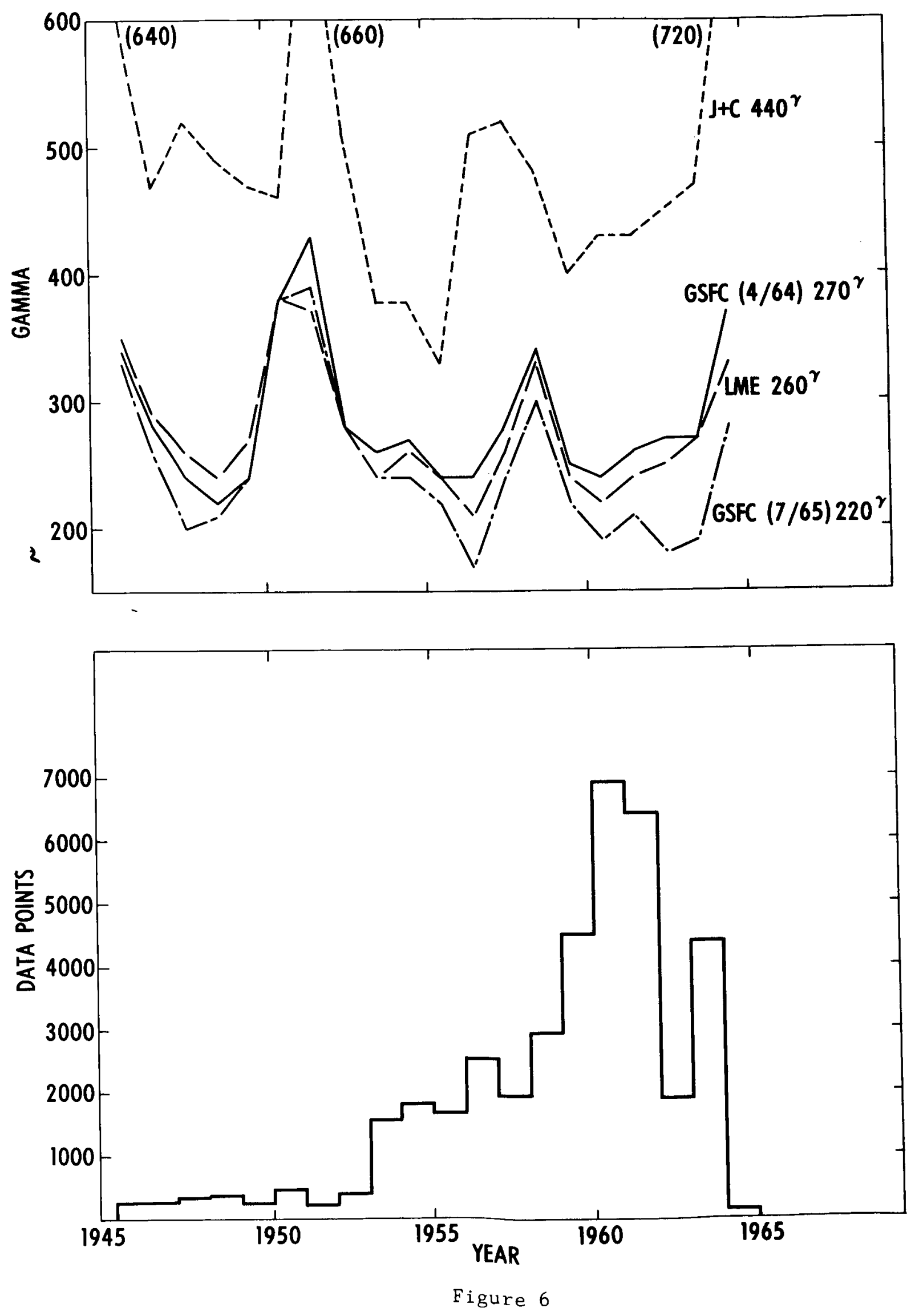
of each curve the RMS for the whole interval 1945-1965. The distribution of data used per year corresponds to a fifth of that available and was obtained simply by using only every fifth data point from a magnetic tape containing all data sequenced in order of longitude. Such a selection procedure gives almost the same results when compared with deviations calculated using all of the available data. One can see that for the twenty years plotted there is about factor of two between the highest and lowest curves. For the data taken over the years 1960-1963 the J+C field errors are growing to about 2.5 times those of the latest field (GSFC $[7 / 65])$. The values for 1964 should not be considered significant since they are based on only 130 observations and are influenced by a Eew large deviations.

Figure 6 shows an unusual periodicity that appears to peak both near solar minimum and maximum. However, if one discounts the 1964 points due to a lack of data there is still the large maximum for 1951-1952. On closer inspection of the data it appears that this is also due to the relatively small amount of data (230 and 240 observations respectively) and arises from large deviations of the surface $Z$ measurements. These $Z$ data are frequently found in error with large inaccuracies. The maximum of all the curves near the sunspot maximum years of the IGY (1957-1958) cannot be explained so easily and might indeed reflect the greater incidence of magnetic disturbance in the data.

Of course the use of RMS deviations does not completely characterize the extent to which the fields fit the observed data. As shown in the 
previous paper, sample detailed deviations of data over various ship and aircraft survey tracks systematic deviations from the GSFC(64) field of wavelengths up to a few thousand kilometers. We have not yet tested the GSFC(7/65) field to see whether these systematic deviations have been largely eliminated or whether an even larger number of coefficients may be necessary. One indication that there may be a need for still more parameters is that, whereas the Vanguard 3 data taken by themselves are consistent to $17 \gamma$ (ㅁan and Hendricks, 1964) the residuals from the GSFC(64) field are 50Y and those from the GSFC(7/65) field are still $28 y$.

\section{Conclusions:}

Sisme of the conclusions one may draw from this discussion must be qualitiative since sufficiently accurate observations of the magnetic field within the magnetosphere are not yet available. In considering the mathematical models necessary to derive the magnetic field we have introduced the concept of a forecast verses a definitive field and have shown that both must eventually include al lowance for plasma-field interactions beyond a few earth radii if the computed fields are to match the ambient field well enough to be useful in helping to understand such experimental data as those from trapped particles. Within a few earth radii, where the effect of the core field predominates, the requirements for increased accuracy may also include making allowance for ionospheric currents and, below a few tens of kilometers, estimates of the crustal field.

Our present estimates of the core field are greatly hampered by the very irregular space-time distribution of magnetic survey data. Nevertheless, 
the significant spatial wavelengths in this field appear to require at least a factor of two more parameters than the 48 spherical harmonics previously used. Fitting the measured data themselves instead of data read from magnetic charts has proved a useful approach and produced fields whose errors in regions of good data coverage are only a fraction of those previously obtained.

The presence of a simple external field near the earth's surface was determined but more work will need to be done to assure its reality. It is much smaller than any previous estimates but is of the correct order of magnitude to be supported by physical theory.

The problems of determining or minimizing the effect of neglected parameters or of investigating the mathematical significance of the areas void of data have not yet been solved. The present fits to the core field, although containing a large number of parameters, do not appear to be significantly worse in the sparse data areas than fits using a smaller number of coefficients. In the areas of good data coverage the new field can be shown to have much smaller errors. If the satellite magnetometer experiments planned for the next year are successful it is likely that the sparse data area problem can be quickly solved and that work can begin to include the contributions of external sources to the field models. 


\section{Acknowledgements}

It is a pleasure to acknowledge the significant contributions that are continuing to be made by the personnel at Goddard: S. Hendricks who has organized and processed the survey data and supervised most of the machine computations and W. E. Daniels who has been instrumental in developing some of the statistical procedures. The basic survey data are the result of the international effort of the World Magnetic Survey IQSY and were supplied on magnetic tape as one of the products of the USC\&GS-NASA Cooperative Data Reduction Program. 


\section{References}

Cain, Joseph C., and Shirley J. Hendricks, Comments on the Vanguard 3 magnetic field data and analysis, J. Geophys. Res., 69, 4187-4188, 1964.

Cain, Joseph C., Shirley Hendricks, Walter E. Daniels, Duane C. Jensen, Computation of the main geomagnetic field from spherical harmonic expansions, NASA X-611-64-316, 1964.

Cain, Joseph C., W. E. Daniels, Shirely J. Hendricks, and Duane C. Jensen, An evaluation of the main geomagnetic field, 1940-1962, J. Geophys. Res., 70, $3647-3674,1965$

Davis, T. N., J. D. Stolarik, and J. P. Heppner, Rocket measurements of Sq currents at mid-latitude, NASA X-612-65-226, 1965 (to be published in J. Geophys. Res.)

Hoffman, R. A., and P. A. Bracken, Magnetic effects of the quiet time proton belt, J. Geophys. Res. 70, 3541-3556, 1965.

Jensen, D. C., and J. C. Cain, An interim geomagnetic field, (Abstract), I. Geophys. Res., $67,3568-3569,1962$.

Kahle, A. B., J. W. Kerr, and E. H. Vestine, Spherical harmonic analyses for the spheroidal earth, Rand Memorandum RM-4360-NASA, 1964.

Leaton, B. R., Remarks on paper by P. F. Fougere 'Spherical harmonic Analysis,' J. Geophys. Res. 6으, 5902, 1963.

Mead, Gilbert D., Deformation of the geomagnetic field by the solar wind, J. Geophys. Res., $\underline{6}$, $1181-1195,1964$.

Ness, Norman F., Measurements of the magnetic fields in interplanetary space and the magnetosphere, NASA X-615-65-352, 1965 .

Sugiura, Masahisa, Hourly values of equatorial Dst for the IGY, Annals of the International Geophysical Year, 35, part I, 9-45, 1964. 